\title{
MAN.04 - Institutional policies: how the market prepares, documents and discloses its guidelines
}

\author{
Chayana Leocadio da Silva ${ }^{1 *}$; Francisco Elton Ribeiro ${ }^{1}$. \\ 1 Fiocruz/Bio-Manguinhos.
}

Introduction: Institutional policies are guidelines established by organizations to achieve their objectives. Therefore, they should be followed by people who are responsible for managing the business and its relationships with stakeholders. Organizations without clear guidelines tend to incur in serious regulatory, legal, cost-enhancing and quality reduction detrimental impacts.

Objective: Evaluate how the market establishes and discloses its guidelines of conduct to achieve internal strategic objectives through institutional policies.

Methodology: For this study, 12 large institutions were analyzed, of which 5 were public. For this purpose, general and specific questionnaires were sent, such as: "What is the current structure or hierarchy of documents established in the Organization?", "Is there any formalized classification for Institutional Policies as to its scope or comprehensiveness?", Are there formal criteria for assessing whether the policy is effectively contributing to the company's overall objective and values?", "What are the topics covered in Institutional Policies"', How is the process of establishing Institutional Policies (elaboration, review, approval ), "Is there a document that describes the sanctions and penalties, in case someone does not follow the Institutional Policies?". And, from the answers, an analysis and diagnosis report was prepared.

Results: $30 \%$ of the companies surveyed reported that they identified the need to establish institutional policies by a legal requirement or because it was a critical issue or a good market practice. The topics most covered were: risks, internal controls and compliance; human resources; relationship with the market; Quality, Occupational Safety, Environment and Occupational Health; information security. No company surveyed organizes their policies from a Value Chain assessment. As for the process of elaboration, validation and dissemination, 8 companies have the practice to elaborate their policies by the functional areas; generally submit for review by an area with an impartial role; and the approval is performed top management. 5 of them disclose their policies through the organization's website, thus providing broad access to the population. Only 1 of them controls and monitors compliance with policies through performance indicators. And in none of them there is a document describing the sanctions and penalties, applicable in case some employee does not follow the guidelines described in institutional policies. In this case, the external regulations and / or applicable legislation are followed.

Conclusion: Through policies, the top management notifies the employees their behaviors intentions within the organization. For organizations that look after the transparency of information or where such standards are mandatory by regulatory bodies, these guidelines are also disclosed to consumers / citizens.

Keywords: institutional policies; guidelines, research 